LBL-36483

UC-414

CBP-115

\title{
Analysis of Optical Stochastic Cooling Including Transverse Effects
}

K.-J. Kim and A.V. Zege

\author{
Accelerator and Fusion Research Division \\ Lawrence Berkeley Laboratory \\ University of California \\ Berkeley, California 94720
}

\section{RECEIVED \\ AUG 171995 \\ OSTI}

May 1995

DISTFIBUTION OF THIS DOCUMENT IS UNLINATED

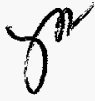

This work was supported by the U.S. Department of Energy under Contract No. DE-AC03-76SF00098. 


\section{DISCLAIMER}

This report was prepared as an account of work sponsored by an agency of the United States Government. Neither the United States Government nor any agency thereof, nor any of their employees, make any warranty, express or implied, or assumes any legal liability or responsibility for the accuracy, completeness, or usefulness of any information, apparatus, product, or process disclosed, or represents that its use would not infringe privately owned rights. Reference herein to any specific commercial product, process, or service by trade name, trademark, manufacturer, or otherwise does not necessarily constitute or imply its endorsement, recommendation, or favoring by the United States Government or any agency thereof. The views and opinions of authors expressed herein do not necessarily state or reflect those of the United States Government or any agency thereof. 


\section{DISCLAIMER}

Portions of this document may be illegible in electronic image products. Images are produced from the best available original document. 
To be published in the Proceedings of the 1995 Particle Accelerator Conference

\title{
ANALYSIS OF OPTICAL STOCHASTIC COOLING INCLUDING TRANSVERSE EFFECTS *
}

\author{
K.-J. Kim and A.V. Zege, Lawrence Berkeley Laboratory, Berkeley, CA, 94720 USA
}

\begin{abstract}
The phase space area plays an important role in determining the performance of optical stochastic cooling. Specifically, the number of samples in the beam consists of three factors corresponding to three dimensions, the factor in each dimension being given by the ratio of the total phase space area in that dimension to the radiation wavelength.
\end{abstract}

\section{INTRODUCTION}

In microwave stochastic cooling [1], the maximum cooling rate is limited by the number of particles $n_{s}$ in a socalled "sample", defined to be the subset of particles that. can interact with a given particle through the pick-upamplifier-kicker system. The signal from the particles in the sample, other than that from the particle itself, contribute to the heating term. A smaller value of $n_{s}$ therefore leads to a faster cooling. If the bandwidth of the pick-upamplifier-kicker system is denoted by $W, n_{s}$ is given by the well-known relationship

$$
n_{s}=n \frac{1}{F} \text {. }
$$

Here, $n$ is the total number of the particles in the beam, $F=T W$, and $T$ is the time duration of the beam. Equation (1) is the statement that the signal due to a given particle appearing at the kicker lasts about $1 / W$, and hence only particles within that temporal distance can interact. with the given particle. The quantity $F$ is referred to as the number of the samples contained in the beam.

Recently, optical stochastic cooling was proposed to take advantage of the larger bandwidth possible with amplifiers in the optical wavelength region, which could be as high as $10^{13}-10^{14} \mathrm{~Hz}$ [2]. Thus the sample size $n_{s}$, and hence the cooling time, would be greatly reduced. A quadrupole undulator and a normal dipole undulator respectively play the role of the pick-up and the kicker in the original scheme in reference[2]. However, the requirement on the beam emittance in that scheme turns out to be very stringent, being about $\lambda \sigma_{\delta}$ or less, where $\lambda$ is the wavelength of the radiation used for the cooling and $\sigma_{\delta}$ is the rms relative energy spread in the bearn. The transit time scherne of optical stochastic cooling was proposed to avoid this limitation [3] In this scheme, normal undulators are employed for both the pick-up and the kicker, and the information on particle coodinates is transmitted via the coordinate-dependent. transit time of the particle trajectory between the pick-up and the kicker undulators.

\footnotetext{
*This work was supported by the U.S. Department of Energy under Contract No. DE-AC03-76SFO0098.
}

The purpose of this paper is to study the effect of the transverse structure of the radiation field on the cooling performance. To this end, we analyze the transit time scheme of the optical stochastic cooling in detail, using an approximate model for the undulator radiation to represent the space-time dependence of the undulator field [4]. Our main finding is that the number of samples in the beam $F$ can be written as

$$
F=F_{L} F_{T_{x}} F_{T_{y}},
$$

where $F_{L}$ and $F_{T x}\left(F_{T y}\right)$ are referred to respectively as the number of the longitudinal and the transverse samples in the $x(y)$-direction. We find that the number of the samples in each dimension is given by the ratio the total phase space area to the coherent phase space area $\lambda$.

The relevance of the transverse structure of the radiation field in optical stochastic cooling was first pointed out in reference[3] based on an intuitive argument. However, the expression for the number of the transverse samples conjectured there-the ratio of the beam size to the coherent. mode size- is valid only in the limit of parallel beam.

The expression for $F_{L}$ as the ratio of the total longitudinal phase space area to the coherent phase space area $\lambda$ reduces the usual result. $T W$ in the long bunch limit. In the short bunch limit, however, $F_{L}$ becomes the ratio of the bean energy spread to the coherent energy spread. This could have interesting consequences for the cooling of bunched beams.

\section{PARTICLES AND FIELDS}

Let $x_{j}$ and $x_{j}^{\prime}$ be respectively the position and the angle of the betatiron motion of the $\mathrm{jth}$ particle as it passes the center of the pick-up undulator, and $\delta_{j}$ its relative energy deviation. The total transverse coordinates are therefore

$$
x_{P j}=x_{j}+\eta \delta_{j}, x_{P j}^{\prime}=x_{j}^{\prime}+\eta^{\prime} \delta_{j},
$$

where $\eta$ and $\eta^{\prime}$ are respectively the dispersion and its derivative at the center of the pick-up undulator. In the following, we will be mainly concerned with the behavior in the $\mathrm{x}$-direction where the dispersion occurs. The behavior in the $y$-direction will become obvious.

Following the work in [3], we choose the betatron transfer matrix between the centers of the pick-up and the kicker undulators to be $-I$, and the dispersion function at the center of the kicker to satisfy the symmetry relation $\left(\eta_{K}, \eta_{K}^{\prime}\right)=\left(\eta,-\eta^{\prime}\right)$. Thus, the transverse coordinates of the it.t particle in the center of the kicker $\left(x_{K i}, x_{K i}^{\prime}\right)$ are related to those at the pick-up as follows:

$$
x_{K i}=-x_{i}+\eta \delta_{i}, x_{K i}^{\prime}=-x_{i}^{\prime}-\eta^{\prime} \delta_{i} .
$$


The path length of the particle trajectory between the centers of the pick-up and the kicker in the linear approximation is given by

$$
l_{j}=l_{0}+\Delta l_{j}=l_{0}+2\left(\eta x_{P_{j}}^{\prime}+Q \delta_{j}\right) .
$$

Here, $Q=\eta \eta^{\prime}+\int_{0}^{l_{0}} d s D(s) / \rho(s), D(s)$ being the contribution to the dispersion from the dipole magnets and $\rho(s)$ the radius of the curvature of the particle trajectory[3]. In this paper, the betatron focussing in the undulators is neglected so that particle trajectories are straight. The trajectory of an ith particle in the kicker undulator can therefore be written as

$$
x_{K i}(t)=x_{K i}+x_{K i}^{\prime} z_{K i}(t), x_{K i}^{\prime}(t)=x_{K i}^{\prime} .
$$

The quantity $z_{K i}(t)$ in $\mathrm{E}_{\mathrm{l}} .(6)$ is the longitudinal coordinate given by:

$$
z_{K_{i}}(t)=\beta_{i}\left(1+x_{K i}^{\prime 2} / 2\right)\left(c t-s_{i}-\Delta l_{i}\right),
$$

where $\beta_{i}$ is the speed of the ith electron divided by $c, c$ the speed of light, and $s_{i}=c t_{i}$ the distance of the the ith particle behind the reference particle in the pick-up undulator. It is necessary in Eq. (7) to keep the second order term in the expansion of $\cos x_{K_{i}}^{\prime}$ because the leading term is sometimes cancelled out. The constant term $l_{0}$ in the path length does not appear in $\mathrm{E}_{\mathrm{q}}$. (7) because the time $t=0$ is defined to be the moment, when the reference particle passes through the center of the kicker undulator.

To find the field produced by the $j$ th electron $E_{P j}\left(x ; z_{P}\right) \exp i \omega\left(t-t_{j}\right)$, where $\omega=2 \pi c / \lambda=k c$, it is sufficient to note the relation at $z_{P}=0$ (the center of the pickup undulator $), E_{P j}(x ; 0)=\exp \left[i k_{x^{\prime}}^{\prime}\left(x-x_{P j}\right)\right] E_{0}(x ; 0)$, where $E_{0}$ is the field of the reference particle[4]. We will approximate $E_{0}(x ; 0)=\exp \left(-x^{2} / 4 \sigma_{r}^{2}\right)$, where $\sigma_{r}$ is the rms mode size at the waist. The field for $z_{P} \neq 0$ is then obtained by a Fresnel transformation. The resulting expression is further simplified by neglecting the $z_{P}$-dependence in the spotsize and the wavefront curvature. The result is then multipled by the factor $\exp \left[-\left(z-c\left(t-t_{j}\right)\right) / 4 \sigma_{C}^{2}\right]$, where $\sigma_{C} \simeq N \lambda / \sqrt{2 \pi}, N$ being the number of the undulator periods. This factor is introduced to account. for the fall-off the field strength due to the increase of the spotsize as well as the fact that the relative bandwidth of the undulator radiation is about, $1 / N$. In this way, we obtain

$$
\begin{aligned}
& E_{P j}\left(x ; z_{P}, t\right)=E_{0} \exp \left[i k_{z_{P}}\left(1-x_{j}^{\prime 2} / 2\right)-i k\left(c t-s_{j}\right)-\right. \\
& \left.\frac{\left(z_{P}-c t+s_{j}\right)^{2}}{4 \sigma_{C}^{2}}+i k x_{j}^{\prime}\left(x-x_{j}\right)-\frac{\left(x-x_{j}-z_{P} x_{j}^{\prime}\right)^{2}}{4 \sigma_{r}^{2}}\right]
\end{aligned}
$$

In deriving Eq.(8), we have assumed that the undulator bandwidth, $1 / N$, is much lager than both the relative energy spread of the particle beam $\sigma_{\delta}$ and the line width due to the angular spread, $\gamma^{2} x^{\prime 2}$, where $\gamma$ is the relativistic factor and $x^{\prime}$ is the angle at the pick-up or kicker undulator.

We choose the optical ABCD matrix for the pick-upamplifier-kicker system to be the same as that of the betatron transfer matrix, $-I$, with an an amplification factor $g$. The field at the kicker $E_{K j}$ is then related to that from the pick-up by $E_{K j}(x, z, t)=g E_{P_{j}}\left(-x, z, t-l_{0} / c-\Delta s_{j} / c\right)$. Here, $z$ is the longituninal coordinate measured from the center of the kicker undulator.

\section{COOLING AND HEATING TERMS}

In the kicker undulator, the energy of a particle becomes modified due to the coupling of its transverse motion in the undulator magnetic field and the transverse electric field of the amplified undulator radiation from the pick-up undulator. The change in the relative energy of the ith particle is

$$
\begin{aligned}
\Delta \delta_{i}=e \frac{g K_{u}}{2 m c^{2} \gamma^{2}} & I n \sum_{j} \int d t E_{P_{j}}\left(-x_{K i}(t), z_{K i}(t), t-\Delta t_{i}\right) \\
& \times \exp \left(i k_{u} z_{K i}(t)-z_{K i}^{2} / 4 \sigma_{L}^{2}\right) .
\end{aligned}
$$

The complex notation for the field is used here with $I m$ implying the imaginary part, and $\varepsilon$ is the particle charge, $m$ its mass, $K_{u}$ the undulator deflection parameter, $k_{u}=$ $2 \pi / \lambda_{u}, \lambda_{u}$ the undulator period length, and the trajectory of the ith paricle is specified by Eq.(6) and Eq.(7). We are assuming that the undulator strength is tapering out as a Gaussian function with an rms length $\sigma_{L} \simeq L / \sqrt{2 \pi}$. Also note the relation $\sigma_{C}=(1-\beta) \sigma_{L} \simeq \sigma_{L} / 2 \gamma^{2}$. Performing the $t$-integral, we obtain

$$
\Delta \delta_{i}=G I m\left(\Phi_{i i}+\sum_{j \neq i} \Phi_{i j}\right),
$$

where $G=e g K_{u} E_{0} L / 2 m c^{2} \gamma^{2}$, and

$$
\begin{gathered}
\Phi_{i j}=\frac{\sigma_{r}}{\Sigma_{i j(2)}} \exp \left\{-\frac{k^{2} \sigma_{L}^{2} \sigma_{r}^{2} x_{i j}^{\prime 4}}{8 \Sigma_{i j(2)}^{2}}-i k \Delta l_{i}-i k\left(s_{i}-s_{j}\right)\right. \\
\left.-\frac{\left[\left(x_{i j}-x_{i j}^{\prime}\left(s_{i}-s_{j}\right) \sigma_{L} / \sigma_{C}\right)^{2}-x_{i j}^{2}-\left(s_{i}-s_{j}\right)^{2} \sigma_{r}^{2} / \sigma_{C}^{2}\right]}{8 \Sigma_{i j(2)}^{2}}\right\} \\
\times \exp \left(i \varphi_{i j}\right)
\end{gathered}
$$

Here

$$
\begin{gathered}
\Sigma_{i j(2)}=\sqrt{\sigma_{r}^{2}+x_{i j}^{\prime 2} \sigma_{L}^{2} / 2}, \Sigma_{i j(1)}=\sqrt{\sigma_{r}^{2}+x_{i j}^{\prime 2} \sigma_{L}^{2}} \\
l_{i}=\Delta t_{i} / c, \quad s_{i}=t_{i} / c, \quad x_{i j}=x_{K i}+x_{P j}=-x_{i}+x_{j}+\eta\left(\delta_{i}+\delta_{j}\right) \\
x_{i j}^{\prime}=x_{K i}^{\prime}+x_{P j}^{\prime}=-x_{i}^{\prime}+x_{j}^{\prime}+\eta\left(-\delta_{i}+\delta_{j}\right)
\end{gathered}
$$

The phase term $\varphi_{i j}$ in the last factor in Eq.(11) is a real quantity with the property $\varphi_{i i}=0$ and $\left|\varphi_{i j}\right| \ll\left|k\left(s_{i}-s_{j}\right)\right|$ for $i \neq j$. The explicite expression of such a phase term will not be important for the following calculations. The change in the average value of $\delta_{i}^{2}$ is then

$$
\left\langle\Delta \delta_{1}^{2}\right\rangle=2 G \operatorname{Im}\left\langle\delta_{1} \Phi_{11}\right\rangle+n\left(G^{2} / 2\right)\left\langle\Phi_{12} \Phi_{12}^{*}\right\rangle .
$$

Here the angular brackets imply taking the average with respect to the variables $s_{i}, \delta_{i}, x_{i}$, and $x_{i}^{\prime}$. We assume that the 
distribution function in these variables can be factorized into Gaussian functions in each variable, with rms widths $\sigma_{z}, \sigma_{\delta}, \sigma_{x}$, and $\sigma_{x^{\prime}}$. Since all particles are equivalent, we have arbitrarily chosen two indices, $i=1$ and $j=2$. The first term in the above is the cooling term. In obtaining the heating term, the second term in $E_{1} .(12)$, we have used the fact that the average of the quantity $\Phi_{1 j} \Phi_{1 j}^{*}$, vanishes unless $j=j^{\prime}$ because of the large, random phase $k\left(s_{j}-s_{j^{\prime}}\right)$.

The cooling term can be evaluated in a closed form as follows:

$$
\begin{gathered}
2 G I m<\delta_{1} \Phi_{11}>=-\frac{4 k G Q \sigma_{\delta}^{2}}{\left(1+2 \eta^{2} \sigma_{\delta}^{2} / \sigma_{r}^{2}\right)^{3 / 2}} \\
\exp \left\{-2 k^{2}\left[\sigma_{x}^{2} \eta^{2}+\frac{Q^{2} \sigma_{\delta}^{2}}{1+2 \eta^{2} \sigma_{\delta}^{2} / \sigma_{r}^{2}}\right]\right\} .
\end{gathered}
$$

This reproduces the expression derived in reference[3] when $\eta \sigma_{\delta} \ll \sigma_{r}$.

The heating term can be written as $n_{s} G^{2} / 2$, where $n_{s}=\left\langle\Phi_{12} \Phi_{12}^{*}\right\rangle$ is the number of particles in a sample for optical stochastic cooling. The averaging with respect to variables $s_{1}$ and $s_{2}$ involves Gaussian integrals. Assuming for simplicity that $\sigma_{z} \gg \sigma_{C}$, the result is

$$
\begin{gathered}
\left\langle\Phi_{12} \Phi_{12}^{*}\right\rangle=\sigma_{C} / \sigma_{z} \times \\
\left\langle\frac{\sigma_{r}^{2}}{\Sigma_{12(1)} \Sigma_{12(2)}} \exp \frac{-1}{4}\left\{\frac{x_{12}^{2}}{\Sigma_{12(1)}^{2}}+\frac{x_{12}^{2}}{\Sigma_{12(2)}^{2}}+\frac{k^{2} \sigma_{r}^{2} \sigma_{L}^{2} x_{12}^{\prime 4}}{\Sigma_{12(2)}^{2}}\right\}\right\rangle .
\end{gathered}
$$

The rest of the averaging will be carried out approximately by regarding the variables $x_{12}$ and $x_{12}^{\prime}$ to be independent with Gaussian distributions of widths $\Sigma_{x}$ and $\Sigma_{x^{\prime}}$, respectively. These quantities will be roughly given by $\Sigma_{x}^{2} \approx 2 \sigma_{x}^{2}+2 \eta^{2} \sigma_{\delta}^{2}$ and $\Sigma_{x^{\prime}}^{2} \approx 2 \sigma_{x^{\prime}}^{2}+2 \eta^{2} \sigma_{\delta}^{2}$. Performing

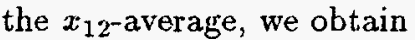

$$
\left\langle\Phi_{12} \Phi_{12}^{*}\right\rangle=\frac{\sigma_{C}}{\sigma_{z}}\left\langle\frac{\sigma_{\tau}^{2} \exp \left[-\left(k \sigma_{L} \sigma_{\tau} x_{12}^{\prime 2} / 2 \Sigma_{12(2)}\right)^{2}\right]}{\Sigma_{12(2)} \sqrt{\Sigma_{12(1)}^{2}+\Sigma_{x}^{2}}}\right\rangle .
$$

The final average in the variable $x_{12}^{\prime}$ can be estimated approximately as follows: First, we assume that the Rayleigh length of the undulator radiation is about $\sigma_{L}$ so that

$$
\sigma_{r} \approx \sqrt{\sigma_{L} / 2 k}, \sigma_{r^{\prime}} \approx 1 / \sqrt{2 k \sigma_{L}}
$$

The exponential factor in Eq.(15) can then be written as $\exp \left(-\xi^{2} / 4(1+\xi)\right)$, where $\xi=\sigma_{L}^{2} x_{12}^{\prime 2} / 2 \sigma_{r}^{2}$. Thus, the average is dominated by the region $\xi \leq 1$. For a rough estimate, therefore, we may set $\xi \approx 0$ everywhere except the exponential function, which we replace by $\exp \left(-\xi^{2} / 4\right)$. The average of this term can be expressed in terms of the modified Bessel function $K_{1 / 4}$. By examining the result, we obtain the following estimate for the heating term:

$$
\frac{G^{2}}{2} n\left\langle\Phi_{12} \Phi_{12}^{*}\right\rangle \equiv \frac{G^{2}}{2} n_{s} \approx \frac{\sigma_{C}}{\sigma_{z}} \frac{\lambda / 2}{2 \pi \sqrt{\left(\sigma_{r}^{2}+\Sigma_{x}^{2}\right)\left(\sigma_{r^{\prime}}^{2}+\Sigma_{x^{\prime}}^{2}\right)}} .
$$

Note here that the quantity $\lambda / 2$ in the numerator is the coherent phase space area $2 \pi \sigma_{r} \sigma_{r^{\prime}}$. Equation (17) is the main result of our analysis in this paper. We consider the meaning of this result in the next section.

\section{THE ROLE OF PHASE SPACE AREA IN LONGITUDINAL AND TRANSVERSE SAMPLES}

Comparing Eq. (17) and Eq.(1), we see that the number of samples in the beam $F$ for the present case is indeed in the form of $\mathrm{E}_{1} .(2)$, with $F_{L}=\sigma_{z} / \sigma_{C}$, and

$$
F_{T x} \approx \frac{\sqrt{\left(\sigma_{r}^{2}+\Sigma_{x}^{2}\right)\left(\sigma_{r^{\prime}}^{2}+\Sigma_{x}^{2}\right)}}{\sigma_{r} \sigma_{r^{\prime}}} .
$$

This reduces the conjecture of reference [3], $\Sigma_{x} / \sigma_{r}$, in the limit $\beta_{x} \gg \sigma_{L}$, where $\beta_{x}$ is the beta function of the particle beam in the kicker undulator. However, note that Eq. (18) consists of two factors, the size and the angular factors. The angular factor arises from the fact that two particles with angular separation larger than the coherent mode angle do not interact, and therefore can not both belong to the same sample.

It is clear that $n_{s}$ will be further reduced by the number of transverse samples in $y$-direction $F_{T y}$, the expression of which will be similar to that of $F_{T x}$.

The analysis of previous section gives $F_{L} \approx \sigma_{z} / \sigma_{C}$ because we assumed $\sigma_{z} \gg \sigma_{C}$. In analogy to the transverse samples, a more general expression is

$$
F_{L} \approx \frac{2 \pi \sqrt{\left(\sigma_{C}^{2}+\sigma_{z}^{2}\right)\left(\sigma_{C \delta}^{2}+\sigma_{\delta}^{2}\right)}}{\lambda / 2}
$$

where $\sigma_{C \delta}=1 / 2 k \sigma_{C}$ is the coherent bandwidth. In the limit, $\sigma_{z} \gg \sigma_{C}, \sigma_{C \delta} \gg \sigma_{\delta}, F_{L}$ becomes the usual expression $\sigma_{z} / \sigma_{C}$. In the opposite limit, $\sigma_{z} \ll \sigma_{C}, \sigma_{C \delta} \ll \sigma_{\delta}$, we obtain $F_{L}=\sigma_{\delta} / \sigma_{C \delta}$. Thus we find in this case that $a$ faster cooling would be achieved with a narrower bandwidth amplifict. The implication of this conclusion for the cooling of bunched beams will be studied in a future paper.

\section{ACKNOWLEDGEMENTS}

We thank Sasha Zholents for numerous useful discussions.

\section{References}

[1] For a comprehensive review, see D. Möhl, CERN Accelerator School Report No. CERN 87-03, 453(1987).

[2] A.A. Mikhailichenko and M.S. Zolotorev, Phys. Rev. Let.t., $71,4146(1993)$

[3] M.S. Zolotorev, A.A. Zholents, Phys. Rev. E, 50, $3087(1994)$.

[4] K.-J.Kim, SPIE Proceedings, vol 582,2 (1986). For a review, see K.-J.Kim, AIP Conference Proceedings No. $184,565(1989)$. 